\title{
Investigations into Enzymatic Bioconversion to Form Rebaudioside A from Stevioside
}

\author{
Réka Czinkóczky ${ }^{1}$ Áron Németh ${ }^{1 *}$ \\ 1 Department of Applied Biotechnology and Food Sciences, Faculty of Chemical Technology and Biotechnology, \\ Budapest University of Technology and Economics, H-1521 Budapest, P.O. Box 91, Hungary \\ * Corresponding author, e-mail: naron@f-labor.mkt.bme.hu
}

Received: 12 June 2018, Accepted: 26 September 2018, Published online: 24 October 2018

\begin{abstract}
Stevia rebaudiana Bertoni is a perennial shrub from South America that produces steviol glycosides which are 200-300 times sweeter than sugar. Stevioside and rebaudioside A are the main sweetening components of its leaves. Steviol glycosides are diterpenoids whose biosynthetic pathways have four steps in common with gibberellic acid formation. The most important enzyme in the biosynthetic pathway expressed by the gene UGT76G1 is referred to as UDP-glycosyltransferase 76G1. It converts stevioside into rebaudioside $\mathrm{A}$. The former has a bitter aftertaste and is a poorer sweetener but is most abundant. This enzyme can be produced in a next generation recombinant way by Escherichia coli and Saccharomyces cerevisiae. Trichoderma longibrachiatum produces the enzyme $\beta$-1,3-glucanase enzyme, which can perform a transglycosylation between stevioside to gain rebaudioside A. In our study, a full-factorial statistical experimental design that applies different glycosyl donors, temperatures, enzyme-to-substrate ratios and pH's as factors in order to achieve higher Reb A ratios in S. rebaudiana extracts after transglycosylation is reported. The presented statistical design was appropriate to indicate relevant and significant factors, providing a good basis for an upcoming experimental design of a real-world optimization.
\end{abstract}

Keywords

Stevia rebaudiana, stevioside, rebaudioside A, $\beta$-1,3-glucanase, transglycosylation

\section{Introduction}

Stevia rebaudiana (Bertoni) (Fig. 1) is a member of the family Asteraceae. This plant originates from South America, especially Paraguay and Brazil in particular. $S$. rebaudiana is a small, herbaceous, perennial shrub of the family Compositae and native to certain regions of South America. The plant inhabited countries around the Pacific Rim, was cultivated domestically and applied in its raw leaf form. Finally it was processed commercially into sweeteners [1, 2]. The Guarani Indians of South America use $S$. rebaudiana to sweeten their tea. The sweetening components of these plants were described in 1931 [3]. The plant $S$. rebaudiana and its extracts have been used for an extensive period of time in China, Japan, Asia, South America and several countries in Europe. The leaves of $S$. rebaudiana and its highly refined extracts are used as low-calorie sweeteners in Korea, Japan and Brazil [4]. The extracts of S. rebaudiana have been approved by the Food and Drug Administration (FDA) and are generally recognized as safe (GRAS). This plant is gaining significance and is expected to develop into a major source of high potency sweeteners for the developing natural food market. The sweetening of foods with extracts from $S$. rebaudiana extracts can increase the attractiveness of food by enhancing of flavors and odors. Sweeteners based on extracts from $S$. rebaudiana are used in beverages, table-top sweeteners, salty and processed food, cosmetics and various delicacies popular in Japan [5]. Stevioside, one of the steviol glycosides (SG's), has been reported to lower the postprandial blood glucose level of Type 2 diabetics and blood pressure of mildly hypertensive patients [6]. S. rebaudiana is used by diabetics as a form of diet therapy, and its extracts exhibit pharmacological effects such as anti-insulin resistance, the promotion of insulin secretion in addition to antihypertensive and anti-obesity properties [7]. Nowadays the utilization of alternative sweeteners has become a viable option to produce low-calorie or zero-calorie foods due to the growth of the healthy-food industry which intends to reduce the sucrose content of food products by totally or 


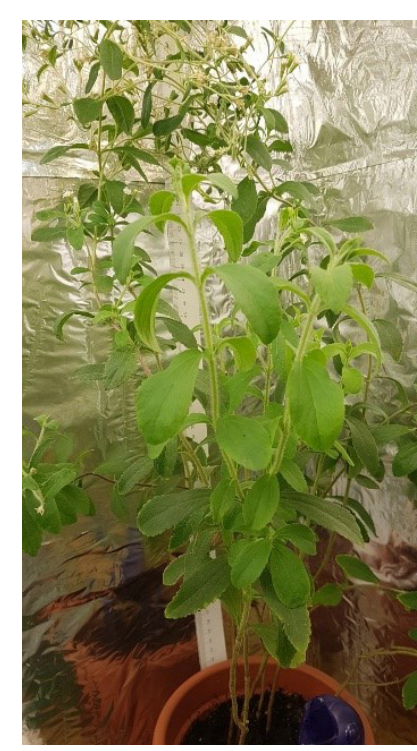

Fig. 1 Stevia rebaudiana

partially replacing sucrose with using alternative sweeteners. The qualities of the food and beverages produced would support the use of $S$. rebaudiana sweetening components instead of sucrose or other sweeteners.

The major components of the plant $S$. rebaudiana are steviol, stevioside and rebaudioside A (Reb A).

The content of SG's is very important for optimal harvesting. The flowers and leaves are often valuable sources of SG.

The content of SG's found in S. rebaudiana varies from 4 to $20 \%$ of the dry weight of leaves depending on the cultivar and growing conditions. Stevioside is the main sweet component in the leaves of $S$. rebaudiana and tastes about 300 times sweeter than sucrose. Stevioside (13-[2-Oß-Dglucopyranosyl- $\alpha$-glucopyranosyl) oxy] kaur-16-en-19oic-acidß-D-glucopyranosyl ester), is a glycoside with a glucosyl and a sophorosyl residue attached to the aglycone of steviol, which consists of a cyclopentanonhydrophenanthrene skeleton [5].

Other minor SG components of lower concentration are steviolbioside, rebaudiosides A-F and dulcoside A [1].

The typical proportions of the major components of the leaves are stevioside (5-10\% of the total dry weight of the leaves), Reb A (2-4\%), Reb C (1-2\%) and dulcoside A (0.4-0.7\%) [5].

The highest total content of the SG's is located in the leaves. The contents of SG's in flowers and stems are 7-8and 12-13-fold lower than in leaves, respectively. In the seeds the amount of the SG's are 2-2.5 times lower the in the flowers. The lowest content of SG's is found in the roots, in fact it is 40 -fold lower than in the leaves [8].

\section{The bioconversion of Stevioside to Rebaudioside A}

The transformation of stevioside to Reb A is one of the most important bioconversions in this field of research area. Reb A has a more pleasant aftertaste and exhibits a higher sweetening effect than stevioside. The stevioside: Reb A ratio is a critical parameter in commercial products as well. Therefore, the aim of this study is to examine the transformation of stevioside to Reb A in primer aqueous $S$. rebaudiana extracts. To select a suitable enzyme, the following three candidates were compared. Firstly, UDPglycosyltransferase UGT76G1 from $S$. rebaudiana catalyzes the conversion of stevioside to Reb A via an one-step glycosylation reaction. This enzyme could be produced by E. coli strain BL21(DE3) [9].

Secondly, hydrolases/transferases of Gibberella fujikuroi can convert stevioside into other relevant SG's, for example Reb A, Reb B and steviolmonoside [10].

Thirdly, $\beta$-1,3-glucanase from Irpex lacteus was applied by Singla et. al (2016) to develop a reaction pathway for an environmentally-friendly transglycosylation catalyzed by an enzyme. This enzyme could catalyze the bioconversion of stevioside to Reb A, and transfers the glucose from curdlan to stevioside (Fig. 2) at an appropriate bond position [11].

The aim of our work was to test different enzymes and glycosyl donors at different temperatures, different $\mathrm{pH}$ 's and enzyme:substrate ratios $(\mathrm{E} / \mathrm{S})$ in order to increase the Reb A to stevioside ratio in $S$. rebaudiana extracts. To select relevant factors, a 2-level full factorial design, that provides a good basis for the experiment design of a real optimization, was generated.

\section{Materials and methods}

For the statistical evaluation, a two-level full factorial design, of which experimental settings and results are listed in Table 1 for each glycosyl donor, was implemented. There were 3 quantitative factors, namely $\mathrm{pH}, \mathrm{E} / \mathrm{S}$ ratio and $\mathrm{T}\left({ }^{\circ} \mathrm{C}\right)$. A qualitative factor referred to as a glycosyl donor, (i.e. water-soluble starch, sucrose, D-trehalose or curdlan) was also examined. Singla and Jaitak [11] used curdlan to conduct this bioconversion of steviol glycoside. 4 center points were made in addition to the $2^{3}$ full factorial design, so in total $\left(2^{3}+4\right) * 4=48$ experiments were conducted.

The applied commercial enzyme preparatum (SigmaAldrich, Germany) contained mainly $\beta$-glucanase from Trichoderma longibrachiatum and exhibited mainly $\beta-1 \rightarrow 3$ / $1 \rightarrow 4$-glucanase activities, but significant xylanase, cellulase, $\beta$-glucosidase, $\beta$-xylosidase, $\alpha$-L-arabinofuranosidase, amylase and protease activities were also present [12]. 


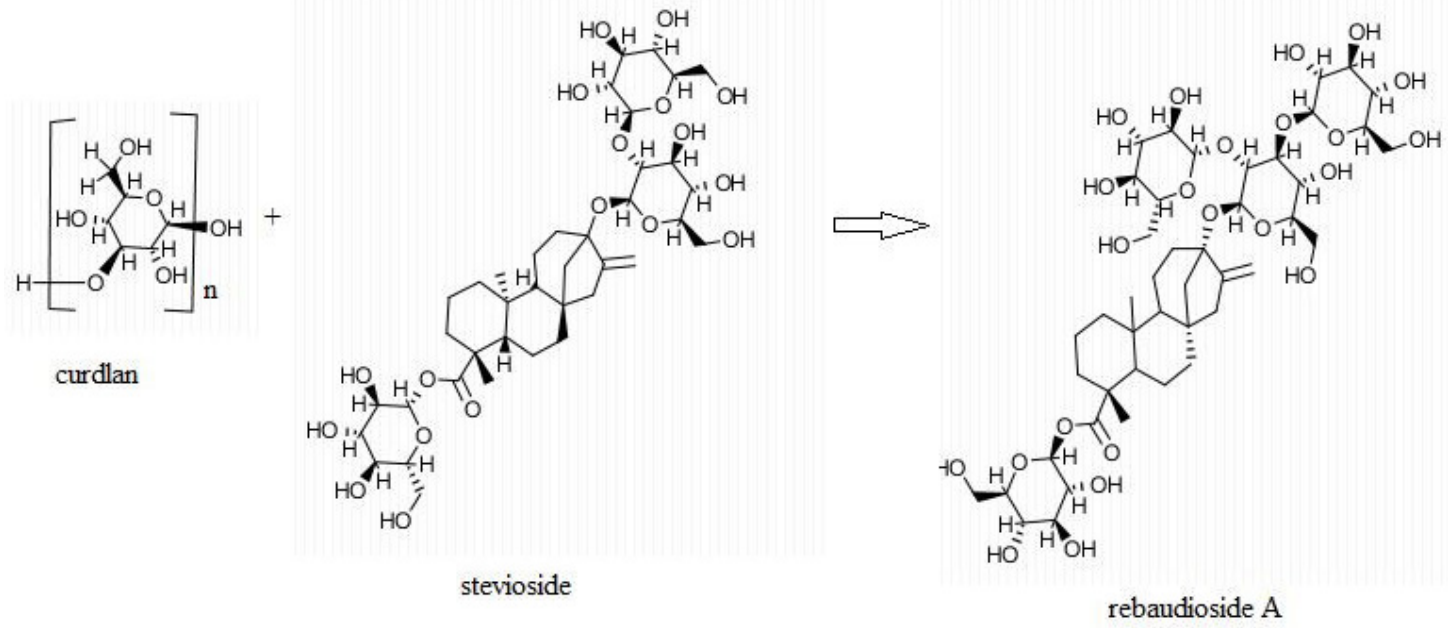

Fig. 2 Transglycosylation of stevioside to Reb A with $\beta$-1,3-glucanase

Table 1 Full factorial design and its results for all glycosyl donors

\begin{tabular}{lccccccc}
\hline order & $\mathrm{pH}$ & $\mathrm{E} / \mathrm{S}$ & $\mathrm{T}^{\circ} \mathrm{C}$ & $\begin{array}{c}\text { DeltaRebA(\%) } \\
\text { water soluble starch }\end{array}$ & $\begin{array}{c}\text { DeltaRebA(\%) } \\
\text { sucrose }\end{array}$ & $\begin{array}{c}\text { DeltaRebA(\%) } \\
\text { D-trehalose }\end{array}$ & $\begin{array}{c}\text { DeltaRebA(\%) } \\
\text { curdlan }\end{array}$ \\
\hline $\mathbf{1}$ & 5 & 3 & 25 & 25.275 & 13.333 & -6.795 & 52.388 \\
$\mathbf{2}$ & 8 & 3 & 25 & 40.861 & 69.991 & 16.637 & -86.765 \\
$\mathbf{3}$ & 5 & 9 & 25 & -56.417 & 0.691 & 7.962 & -41.474 \\
$\mathbf{4}$ & 8 & 9 & 25 & 0.284 & 18.891 & -74.727 & -39.866 \\
$\mathbf{5}$ & 5 & 3 & 45 & 28.480 & -7.808 & 133.572 & 39.577 \\
$\mathbf{6}$ & 8 & 3 & 45 & -7.387 & -35.940 & -40.225 & -14.249 \\
$\mathbf{7}$ & 5 & 9 & 45 & -22.369 & -3.031 & -86.796 & -72.549 \\
$\mathbf{8}$ & 8 & 9 & 45 & -42.717 & 27.103 & 23.738 & 1.664 \\
$\mathbf{9}$ & 6.5 & 6 & 35 & 4.223 & -0.778 & 31.663 & 24.809 \\
$\mathbf{1 0}$ & 6.5 & 6 & 35 & 12.707 & 10.100 & 28.594 & 12.995 \\
$\mathbf{1 1}$ & 6.5 & 6 & 35 & -35.566 & 26.819 & -29.430 & -37.574 \\
$\mathbf{1 2}$ & 6.5 & 6 & 35 & 3.380 & 16.129 & -26.794 & -16.616 \\
\hline
\end{tabular}

The bioconversions took place in $15-\mathrm{ml}$ Falcon conical centrifuge tubes in a temperature-controlled cabinet with a tube rotator equipment (Rotomix, Kutesz, Hungary) (Fig. 3).

The raw material was a pressurized hot-water extract (PHWE) of $S$. rebaudiana leaves. The $S$. rebaudiana extract was collected from the spontaneously dried plant material $(2-3 \mathrm{~mm})$ produced by autoclaving (10 g plant: $200 \mathrm{ml}$ distilled water) in a Tuttnauer 3870 ELV-type laboratory autoclave. After the PHWE $\left(121^{\circ} \mathrm{C}, 20 \mathrm{mins}\right)$ the extract was centrifuged (Hermle Z 200 A, 8 mins., $6000 \mathrm{rpm})$ to remove the solid components.

The $S$. rebaudiana extract and curdlan (from Alcaligenes faecalis, Sigma Aldrich, Germany) were mixed in a 1:2 ratio in a citrate buffer $(0,1 \mathrm{M}$ with different $\mathrm{pH})$ and enzyme preparatum was added according to $\mathrm{E} / \mathrm{S}$ ratio. The solution was mixed and incubated in a Rotomix overnight.
The reaction mixture was brought to room temperature and the enzyme deactivated by boiling it at $100^{\circ} \mathrm{C}$ for 5 mins.

Each sample was measured using high-performance liquid chromatography (HPLC) (ZORBAX Carbohydrate Analysis column) [13] to determine the increase in the peak area of Reb A compared to its initial peak area (deltaRebA(\%)). The mobile phase consisted of a phosphate buffer and acetonitrile in 25:75 volume ratio. The eluent flow rate was $1 \mathrm{ml} / \mathrm{min}$ and a guard column was applied.

The equation of the calibration curve using HPLC was as follows where the peak area is denoted by $\mathrm{y}$ and the concentration of Reb $\mathrm{A}$ by $\mathrm{x}$ : $y=1000000 * x+116103$

The average and standard deviation of retention times were the following for the calibration of Reb A (Fig. 4): RT: $4.565 \pm 0.044 \mathrm{~min}$ 


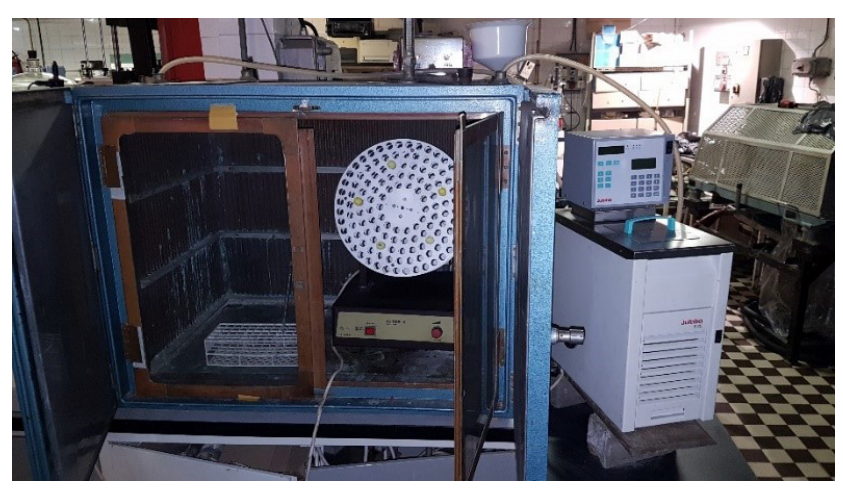

Fig. 3 Tube rolling equipment

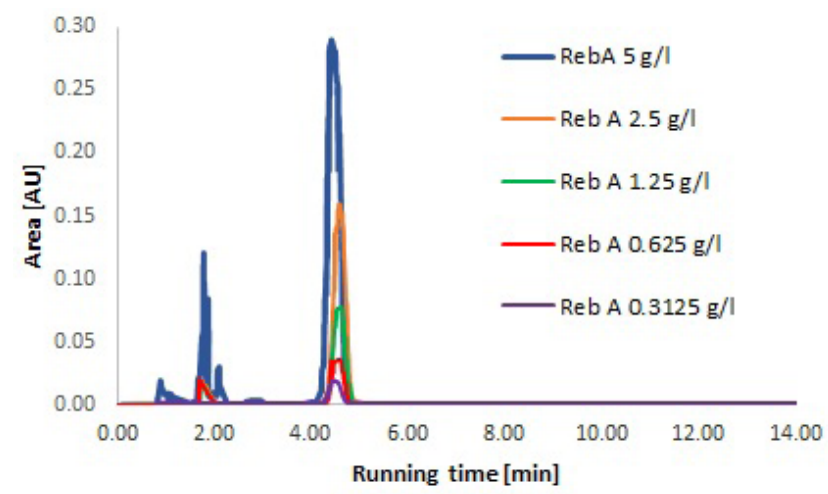

Fig. 4 HPLC overlay diagram: Reb A calibration

Minitab 17 software was used for the statistical evaluations.

\section{Results and discussion}

The changes in the peak area of Reb A using HPLC were measured, which were expressed as deltaRebA(\%) values. The deltaRebA(\%) results showed how the chosen glycosyl donors (water-soluble starch, sucrose, D-trehalose and curdlan) effect the changes in Reb A concentration during the bioconversion.

For each glycosyl donor, the results were first analyzed from a statistical point of view. All residual plots were similar to Fig. 5 indicating that residuum, i.e. the differences between measured and fitted values, exhibit normal distribution, constant variance and are independent.

The first glycosyl donor to be tested was water-soluble starch. In Fig. 6A a Pareto chart is presented, which suggests that the effect of the E/S ratio is more significant than other effect and interactions.

The main effects of tested factors in the case of starch as a glycosyl donor are demonstrated in Fig. 6B, which suggests, that the application of a low-level E/S ratio will increase deltaRebA(\%), while the other factors exhibit

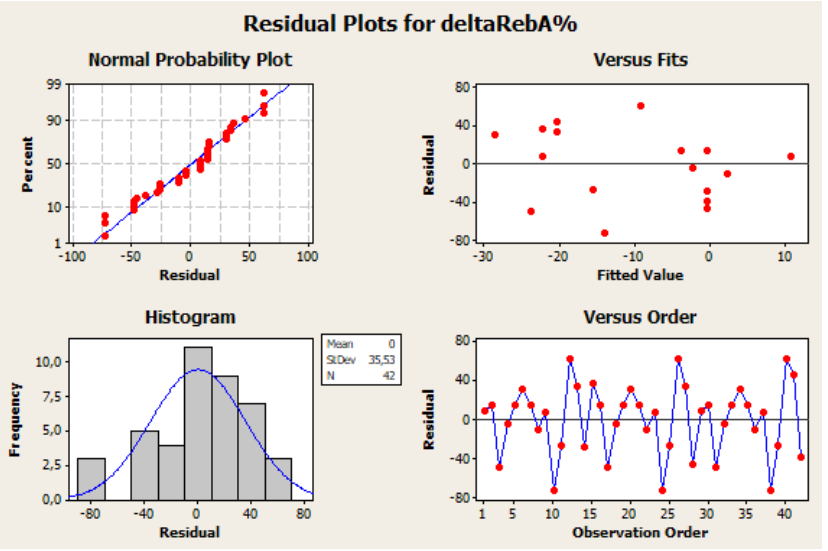

Fig. 5 Residual plots for deltaRebA (\%)

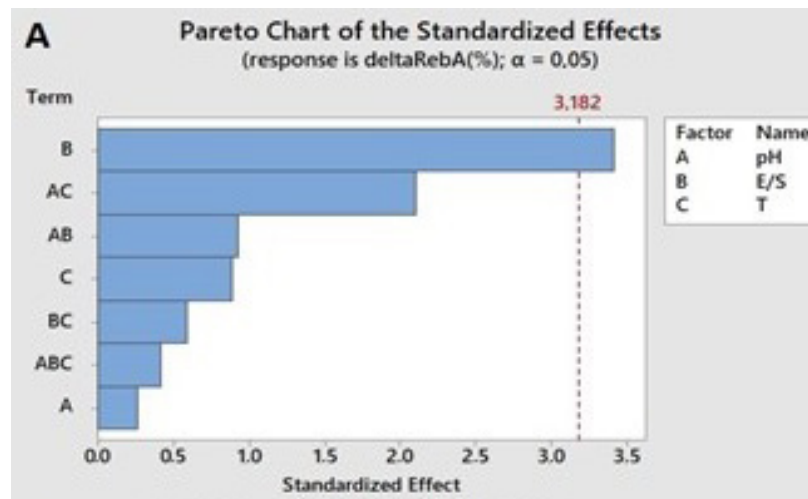

B Main Effects Plot for deltaRebA(\%)
Fitted Means

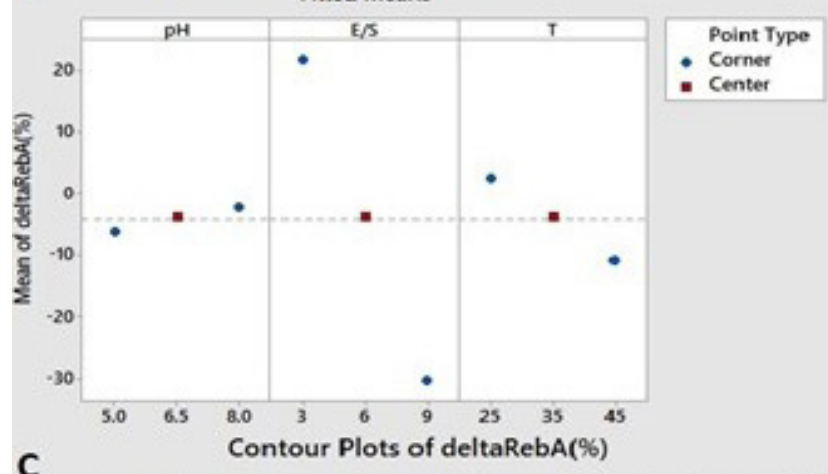

C

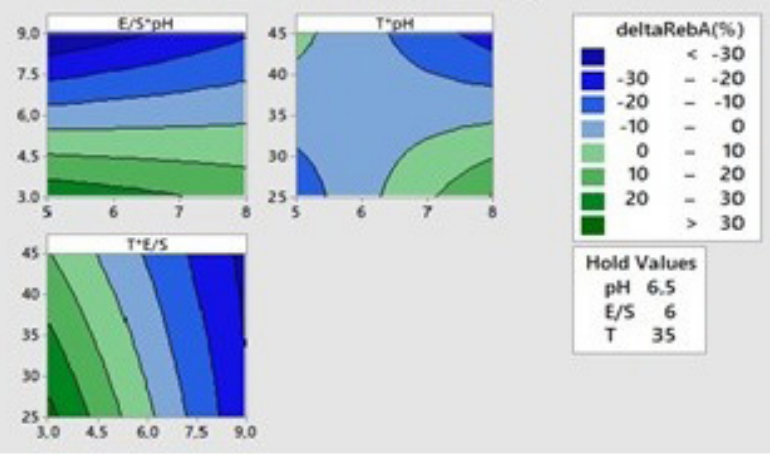

Fig. 6 Glycosyl donor: water soluble starch, A: Pareto Chart, B: Main Effects Plot, C: Contour Plot 
minimal effects. In Fig. 6C the contour plots show how factors influence variation in terms of deltaRebA(\%). Higher and better values are denoted by the dark green area, so low E/S ratios and temperatures are better for the formation of Reb A.

The second glycosyl donor to be examined was sucrose. According to the Pareto chart (Fig. 7A) the effect of temperature and interaction of $\mathrm{E} / \mathrm{S}$ ratio and temperature were significant.

In Fig. 7B the main effects plot shows that the E/S ratio had no effect, but the low temperature and higher $\mathrm{pH}$ have a positive effect on the increase of deltaRebA(\%).

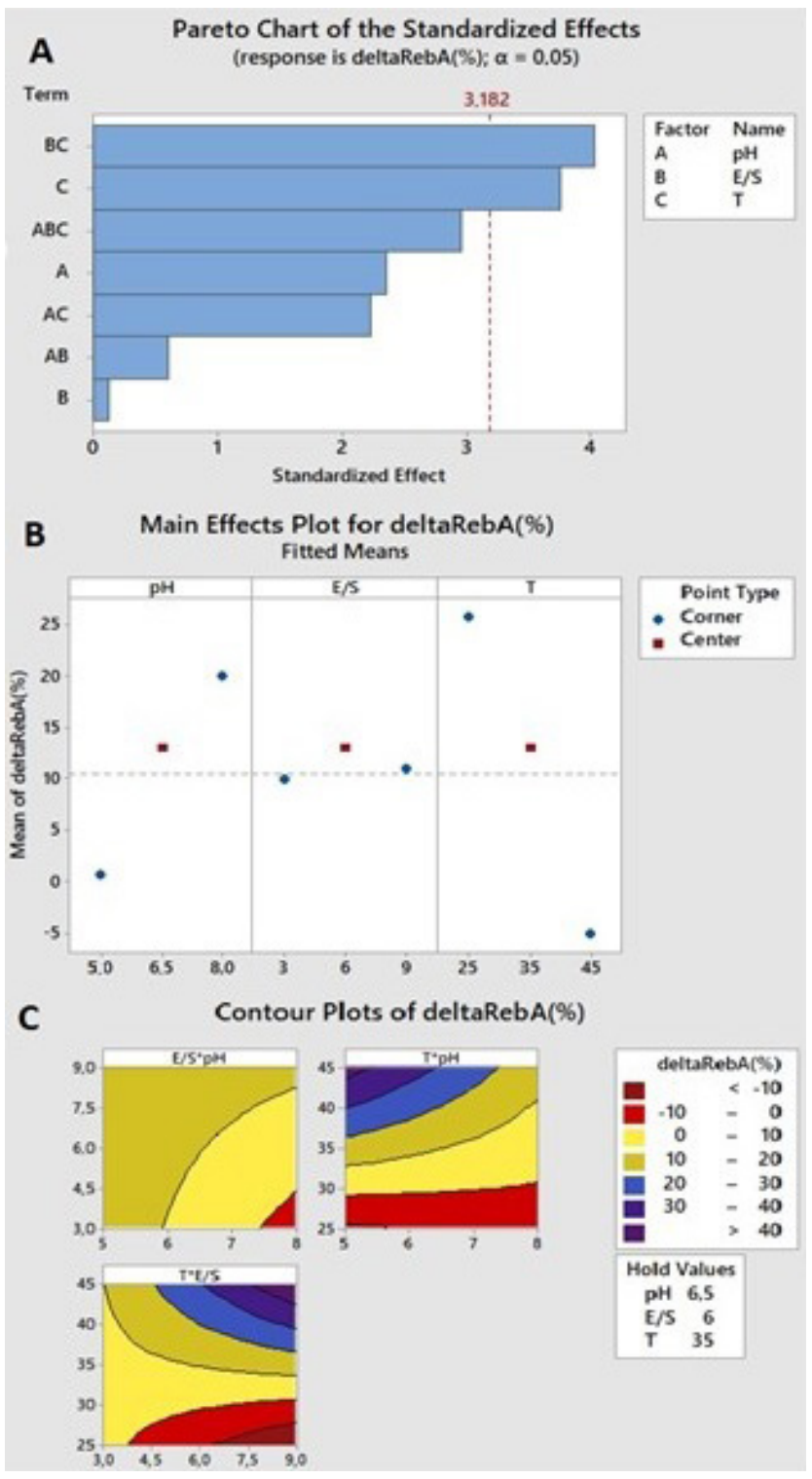

Fig. 7 Glycosyl donor: sucrose, A: Pareto Chart, B: Main Effects Plot, C: Contour Plot
In Fig. $7 \mathrm{C}$ the contour plots illustrate how the factors and their interactions affect deltaRebA(\%). The dark purple area is preferred, i.e. high temperature, low $\mathrm{pH}$ and high $\mathrm{E} / \mathrm{S}$ ratio cause an increase in $\operatorname{deltaRebA}(\%)$.

The third examined glycosyl donor was D-trehalose. The Pareto chart in Fig. 8A shows that the interaction between the $\mathrm{E} / \mathrm{S}$ ratio, $\mathrm{pH}$ and temperature has a significant effect on the increase in $\operatorname{deltaRebA}(\%)$.

In Fig. 8B the main effects plot illustrates that the low levels of $\mathrm{pH}$ and $\mathrm{E} / \mathrm{S}$ ratio in addition to the high temperature have some positive impression on the enhancement of deltaRebA(\%).

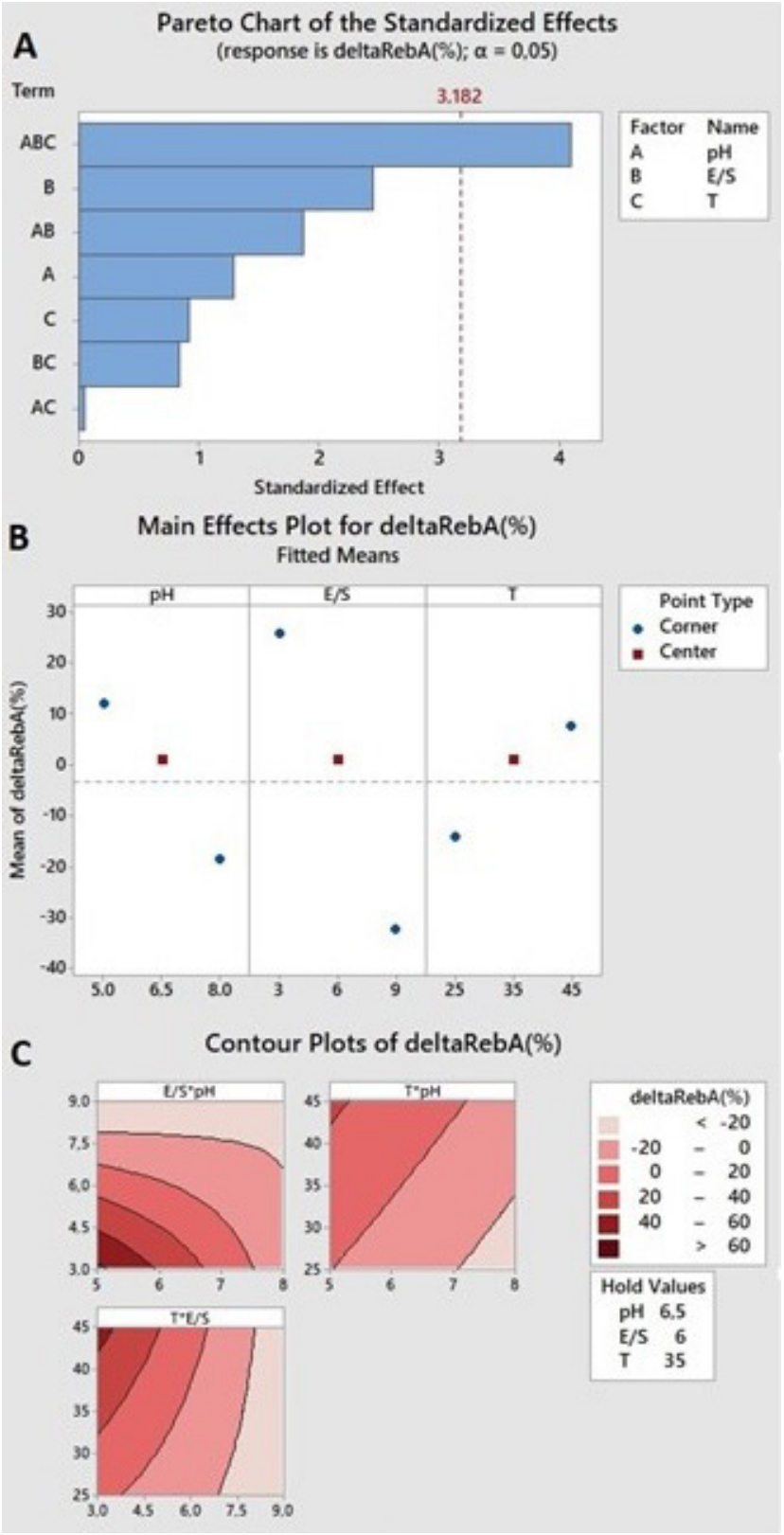

Fig. 8 Glycosyl donor: D-trehalose, A: Pareto Chart, B: Main Effects Plot, C: Contour Plo 
In Fig. 8C the contour plots show how each factor and their interactions alters deltaRebA(\%). The higher values of deltaRebA $(\%)$ are denoted by the dark red regions, therefore, the low $\mathrm{pH}$, low $\mathrm{E} / \mathrm{S}$ ratio and high temperature are preferable for the enhancement of deltaRebA(\%).

In Fig. 9A (glycosyl donor: curdlan) the Pareto chart implies that the interaction between the $\mathrm{pH}$ and $\mathrm{E} / \mathrm{S}$ ratio has a significant effect with regard to increasing deltaRebA(\%) when compared to the other factors and interactions.

The main effects plot in Fig. 9B represents that the lower $\mathrm{E} / \mathrm{S}$ ratio and $\mathrm{pH}$ in addition to the higher temperature have a positive effect in terms of the enhancement of deltaRebA(\%).

In Fig. 9C the contour plots claim that the examined factors and their interactions effect deltaRebA(\%). The dark blue area is the preferable, therefore, a low temperature, low $\mathrm{pH}$ and low $\mathrm{E} / \mathrm{S}$ ratio results in a higher deltaRebA(\%).

\section{Conclusion}

In this paper concerning the bioconversion of steviol glycoside, an attempt was made to identify an alternative glycosyl donor instead of curdlan, which has been frequently reported and applied, to effectively provide a glycosyl group for the enzymatic bioconversion of stevioside to Reb A. A two-level full factorial statistical design was implemented to screen for any significant effect of three factors, namely $\mathrm{pH}, \mathrm{E} / \mathrm{S}$, and $\mathrm{T}^{\circ} \mathrm{C}$.

For the purposes of identifying more detailed effects and correlations, new statistical experimental designs should be drawn up to improve the enzymatic bioconversion of stevioside to rebaudioside A.

\section{Acknowledgement}

We are grateful for the provision of stevia raw materials by Stevia Product Europe Ltd. in Hungary.

\section{References}

[1] Kaur, G., Pandhair, V., Cheema, G. S. "Extraction and characterization of steviol glycosides from Stevia rebaudiana bertoni leaves", Journal of Medicinal Plants Studies, 2(5), pp. 41-45, 2014, [online] Available at: http://www.plantsjournal.com/vol2Issue5/ Issue_sep_2014/10.1.pdf [Accessed: 03 December 2017]

[2] Geuns, J. M. C. "Stevioside", Phytochemistry, 64(5), pp. 913-921, 2003 .

https://doi.org/10.1016/S0031-9422(03)00426-6

[3] Madan, S., Ahmad, S., Singh, G. N., Kohli, K., Kumar, Y., Singh, R., Garg, M. "Stevia rebaudiana (Bert.) Bertoni- A Review", Indian Journal of Natural Products and Resources, 1(3), pp. 267-286, 2010 .

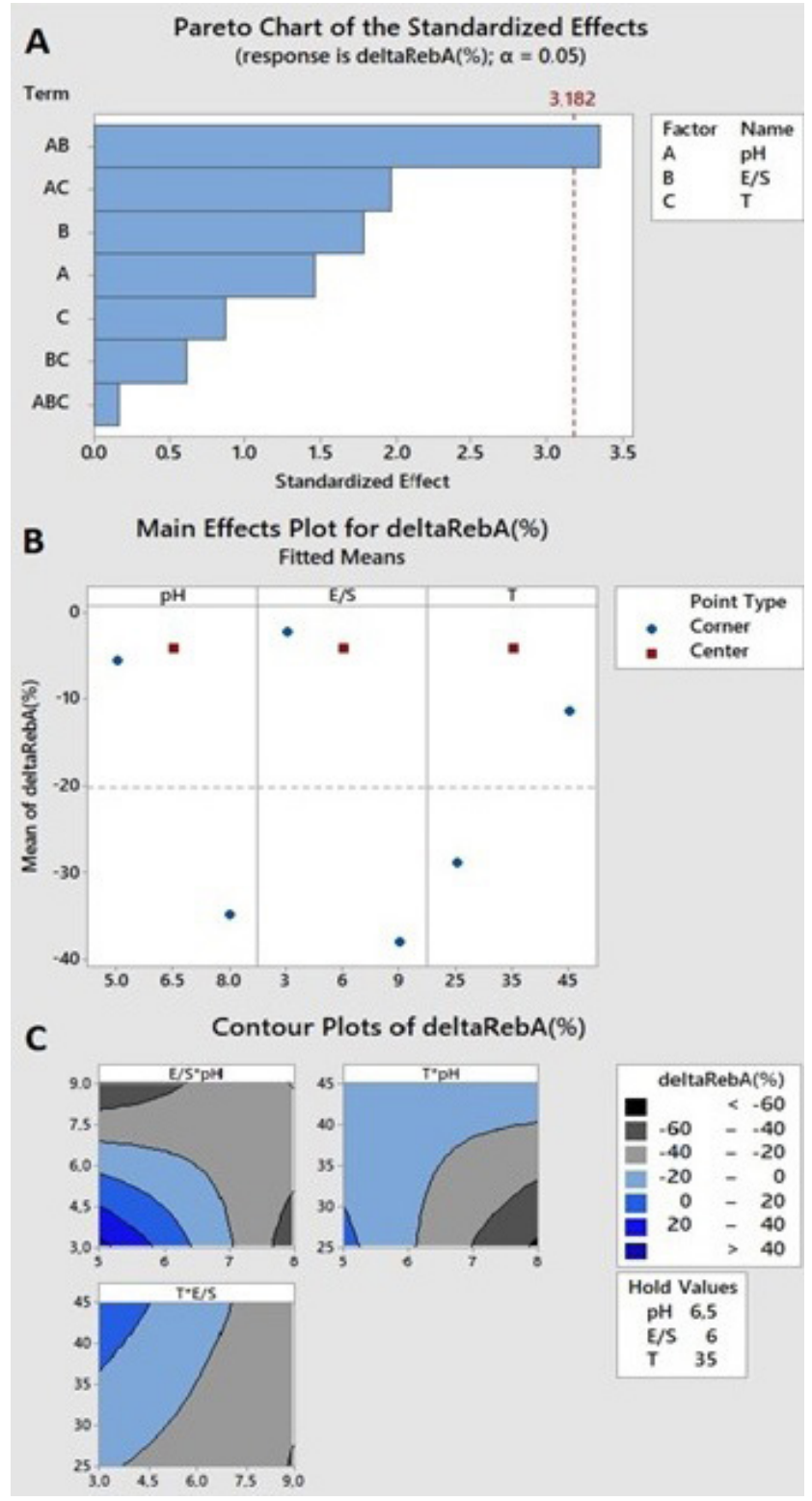

Fig. 9 Glycosyl donor: curdlan, A: Pareto Chart, B: Main Effects Plot, C: Contour Plot

[4] Kinghorn, A. D. "Stevia: The Genus Stevia", 1st ed., CRC Press, Boca Raton, FL, USA, 2001, [online] Available at: https:// www.crcpress.com/Stevia-The-Genus-Stevia/Kinghorn/p/ book/9780415268301 [Accessed: 24 September 2017]

[5] Puri, M., Sharma, D., Tiwari, A. K. "Downstream processing of stevioside and its potential applications", Biotechnology Advances, 29(6), pp. 781-791, 2011.

https://doi.org/10.1016/j.biotechadv.2011.06.006

[6] Assaei, R., Mokarram, P., Dastghaib, S., Darbandi, S., Darbandi, M., Zal, F., Akmali, M., Ranjbar Omrani, G. H. "Hypoglycemic Effect of Aquatic Extract of Stevia in Pancreas of Diabetic Rats: PPAR $\gamma$-dependent Regulation or Antioxidant Potential", Avicenna Journal of Medical Biotechnology, 8(2), pp. 65-74, 2016. 
[7] Dyrskog, S. E. U., Jeppesen, P. B., Colombo, M., Abudula, R., Hermansen, K. "Preventive effects of a soy-based diet supplemented with stevioside on the development of the metabolic syndrome and type 2 diabetes in Zucker diabetic fatty rats", Metabolism, 54(9), pp. 1181-1188, 2005.

https://doi.org/10.1016/j.metabol.2005.03.026

[8] Totté, N., Charon, L., Rohmer, M., Compernolle, F., Baboeuf, I., Geuns, J. M. C. "Biosynthesis of the diterpenoid steviol, an entkaurene derivative from Stevia rebaudiana Bertoni, via the methylerythritol phosphate pathway", Tetrahedron Letters, 41(33), pp. 6407-6410, 2000.

https://doi.org/10.1016/S0040-4039(00)01094-7

[9] Yadav, S. K., Guleria, P. "Steviol Glycosides from Stevia: Biosynthesis Pathway Review and their Application in Foods and Medicine", Critical Reviews in Food Science and Nutrition, 52(11), pp. 988-998, 2012.

https://doi.org/10.1080/10408398.2010.519447

[10] Kuzuyama, T., Takagi, M., Takahashi, S., Seto, H. "Cloning and Characterization of 1-Deoxy-d-Xylulose 5-Phosphate Synthase from Streptomyces sp. Strain CL190, Which Uses both the Mevalonate and Nonmevalonate Pathways for Isopentenyl Diphosphate Biosynthesis", Journal of Bacteriology, 182(4), pp. 891-897, 2000. https://doi.org/10.1128/JB.182.4.891-897.2000
[11] Singla, R., Jaitak, V. "Synthesis of rebaudioside A from stevioside and their interaction model with hTAS2R4 bitter taste receptor", Phytochemistry, 125, pp. 106-111, 2016.

https://doi.org/10.1016/j.phytochem.2016.03.004

[12] Sigma-Aldrich "G4423 SIGMA $\beta$-Glucanase from Trichoderma longibrachiatum", (n.d.), [online] Available at: https://www. sigmaaldrich.com/catalog/product/sigma/g4423?lang=hu\&region=HU [Accessed: 03 March 2018]

[13] Henderson, Jr. J. W., Berry, J. "Isocratic Stevia Sweetener Analysis using Selective ZORBAX Columns", (n.d.), [pdf] Available at: https://www.agilent.com/cs/library/applications/5990-3933EN.pdf [Accessed: 26 January 2018] 